\title{
Constante de mola de molas cerâmicas injetadas a baixa pressão
}

\section{(Spring constant of low-pressure injection molded ceramic springs)}

\author{
R. A. Barbieri, J. E. Zorzi \\ Universidade de Caxias do Sul, Caxias do Sul, RS 95070-560, Brasil \\ jezorzi@ucs.br
}

\begin{abstract}
Resumo
A dificuldade de usinagem de peças cerâmicas já sinterizadas é muito grande, principalmente devido à dureza e fragilidade destes materiais, o que implica em altos custos de produção. Por isso, grandes esforços tem sido feitos no sentido de melhorar os processos de conformação a verde existentes, ou criar novos processos que permitam a obtenção de peças cada vez mais próximas do formato final desejado. Produzir peças cerâmicas com formatos complexos, livres de defeitos, é uma tarefa que implica em grandes dificuldades. Molas cerâmicas possuem formatos extremamente difíceis de serem moldados e, conseqüentemente, atualmente são produzidas comercialmente molas cerâmicas pelo processo de usinagem, geralmente a um custo elevado. Uma alternativa para a produção de molas cerâmicas é a moldagem por injeção em baixa pressão. Para o desenvolvimento de molas cerâmicas para aplicações tecnológicas, é necessário, além de obter peças íntegras e livres de defeitos, aferir algumas de suas propriedades, como a constante de mola. Uma vez que estas molas encontram aplicação em altas temperaturas, torna-se imprescindível realizar a medida da resistência imposta pela mola à deformação elástica em diferentes temperaturas. Para tanto, este trabalho propõem a montagem de um sistema para a medição da constante de mola de molas cerâmicas injetadas a baixa pressão, tanto à temperatura ambiente como em altas temperaturas, usando o método dinâmico da excitação por impulso para medir a frequência de vibração da mola suspensa no interior de um forno. Para ilustrar a aplicação desta técnica são apresentados resultados obtidos para uma mola helicoidal de alumina, da temperatura ambiente até $1100^{\circ} \mathrm{C}$.
\end{abstract}

Palavras-chave: moldagem por injeção em baixa pressão, molas cerâmicas, constante de mola.

\begin{abstract}
The machining of sintered ceramic parts is a difficult process, mainly due to the hardness and brittleness of these materials, which implies in high production costs. Therefore, great efforts have been made to improve the forming processes of green ceramics, or create new processes to obtain the near net shape parts. The production of ceramic parts with complex shapes, free of defects, is a task that involves great difficulties. Ceramic springs exhibits shapes extremely difficult to be molded and therefore are currently commercially produced mainly by machining, a process which is difficult and expensive. An alternative for the production of ceramic springs is by low-pressure injection molding. For the development of ceramic springs for technological applications, it is required in addition to getting parts intact and free of defects, to measure some of its properties, including the spring constant. Since these springs are usually applied at high temperatures, it becomes important to carry out the measurement of resistance imposed by the spring to elastic deformation at different temperatures. Accordingly, in this work we describe the assembly of an experiment for the measurement of the spring constant of low-pressure injection molded ceramic springs, both at ambient temperature and at high temperatures, using the dynamic impulse excitation method to measure the frequency of vibration of a suspended spring inside a furnace. Results are presented for a helical spring of alumina from room temperature to $1100^{\circ} \mathrm{C}$ to illustrate the application of this technique.
\end{abstract}

Keywords: low-pressure injection molding, ceramic springs, spring constant.

\section{INTRODUÇÃO}

Nos últimos anos houve um crescente interesse na fabricação das molas cerâmicas, visando aplicações que exijam resistência à corrosão, tanto em temperatura ambiente como em alta temperatura [1-6]. Molas e enrolamentos cerâmicos têm sido obtidos por usinagem de cilindros, por extrusão de misturas de cerâmicas com polímeros ou pelo processo sol-gel [1-6]. Enquanto o primeiro processo é flexível, mas de custo elevado, nos dois últimos existe a dificuldade de extrair o ligante sem deformar as molas.

As molas cerâmicas possuem a vantagem de suportarem ambientes quimicamente agressivos, pois possuem resistência à corrosão de ácidos e bases e também resistência em temperaturas elevadas. Molas cerâmicas devem possuir elevada tenacidade à fratura, alta dureza, excelente resistência ao desgaste e baixa condutividade térmica [7]. De fato, uma desvantagem das molas metálicas é que elas perdem sua função em altas temperaturas. As molas deformam-se sob a ação de uma carga, armazenando energia, e retornam, ao serem descarregadas, à sua forma inicial, restituindo a energia previamente armazenada [8]. As molas podem ser aplicadas em armazenamento de cargas, amortecimento de choques ou controles dos movimentos. Podem ser 


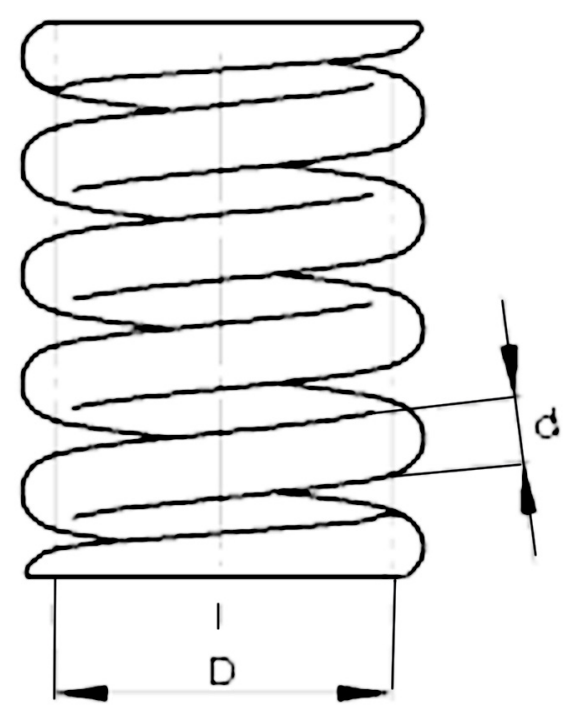

Figura 1: Representação esquemática de uma mola helicoidal [8]. [Figure 1: Schematics of helical spring design [8].]

submetidas a esforços de tração e compressão, flexão e torção. As molas helicoidais, em particular, são utilizadas em esforços de tração e compressão [9]. Em geral, as molas com espaçamento maior entre os fios comprimem mais facilmente, de modo que oferecem uma resistência menor à carga aplicada. Em uma mola que obedece a lei de Hooke, a deflexão deve variar linearmente com a carga aplicada [3]. No caso de molas helicoidais com seção circular, como representado na Fig. 1, a constante de mola é o parâmetro que relaciona a deformação axial de uma mola com a carga aplicada.

As propriedades mecânicas de uma mola dependem da geometria (dimensões) e do módulo de cisalhamento do material que a constitui. Em particular, a constante de mola é dada por

$$
K=\frac{G d^{4}}{8 N_{a} D^{3}}
$$

na qual $K$ é a constante da mola, $G$ é o módulo de cisalhamento do material, $d$ é o diâmetro médio do fio, $N a$ é o número de espiras ativas na mola e $D$ é o diâmetro médio da mola [10] .

Em trabalho anterior, desenvolvemos um procedimento para a produção de molas cerâmicas por moldagem por injeção em baixa pressão (MIBP) [7, 11]. Dois tipos de moldes foram testados. No primeiro, as molas cerâmicas foram produzidas por injeção em um enrolamento helicoidal oco de cobre. As peças a verde foram recuperadas após a remoção das paredes de cobre por tratamento em meio ácido. Na segunda estratégia foi utilizado um molde metálico multipartido revestido com politetrafluoretileno (ptfe) (Fig. 2). Em ambos os casos a intenção era desenvolver um método simples, robusto e de baixo custo para conformar molas helicoidais com perfil circular. Os métodos propostos podem ser utilizados para a produção de molas de cerâmicas avançadas para uso em elementos de aquecimento e dispositivos elétricos e mecânicos, por exemplo. A qualidade das peças cerâmicas sinterizadas foi avaliada por medidas de densidade e de dureza Vickers. As curvas de tensão-deformação para as molas cerâmicas foram obtidas de testes de compressão em uma máquina universal de ensaios, em temperatura ambiente. Os pós cerâmicos usados foram alumina $\left(\mathrm{Al}_{2} \mathrm{O}_{3}\right)$ e zircônia parcialmente estabilizada com ítria $\left(3 \mathrm{Y}-\mathrm{ZrO}_{2}\right)$. As molas sinterizadas de alumina e de zircônia foram obtidas com densidades que variaram de $96 \%$ a $99 \%$ do valor teórico. Nos testes mecânicos, as molas suportaram deformações axiais de $10 \%$ antes da falha $[7,11]$.

A presença de defeitos, como poros e imperfeições na superfície, pode reduzir consideravelmente a resistência mecânica de molas cerâmicas [12, 13]. Quando submetidas a altas temperaturas estes defeitos tendem a se agravar e, além disso, ocorre a diminuição da resistência mecânica como um todo. Produzir molas cerâmicas com formatos complexos livres de defeitos é uma tarefa difícil, pois as molas cerâmicas possuem formatos extremamente difíceis de serem moldados. De fato, o longo comprimento do fio aumenta a possibilidade da retenção de bolhas de ar oriundas do processo de injeção, enquanto que a grande razão superfície/volume torna as peças especialmente suscetíveis aos efeitos dos defeitos superficiais. Para o desenvolvimento de molas cerâmicas para aplicações tecnológicas, é necessário que, além de obter peças íntegras e livres de defeitos, seja possível medir suas propriedades, como a constante de mola em alta temperatura ou em ambientes quimicamente agressivos, o que é difícil de realizar em uma máquina universal de ensaios mecânicos.

Neste trabalho será descrito um procedimento simples que permite a medição da constante de mola de molas cerâmicas injetadas em baixa pressão, desde a temperatura ambiente até $1100{ }^{\circ} \mathrm{C}$. Este procedimento é baseado na medida da frequência de vibração longitudinal da mola, suspensa por um fio no interior de um forno. A constante de mola é então obtida usando uma expressão simples derivada da teoria da elasticidade.

\section{MATERIAIS E MÉTODOS}

\section{Matéria-prima e moldagem das molas}

Foi utilizada como matéria-prima alumina $\left(\mathrm{Al}_{2} \mathrm{O}_{3}\right)$ A-1000SG (Almatis Inc., Leetsdale, PA, EUA), como recebida. A composição segundo o fabricante é $\mathrm{Al}_{2} \mathrm{O}_{3} 99,8 \%$, $\mathrm{N}_{2} \mathrm{O} 0,07 \%, \mathrm{SiO}_{2} 0,03 \%, \mathrm{Fe}_{2} \mathrm{O}_{3} 0,02 \%, \mathrm{CaO} 0,02 \%$ e $\mathrm{MgO}$ $0,04 \%$; área superficial $8,4 \mathrm{~m}^{2} / \mathrm{g}$; porcentagem que passou pela peneira 325 mesh 99,9\%; densidade a verde (5000 psi) $2,14 \mathrm{~g} / \mathrm{cm}^{3}$; densidade sinterizada $3,85 \mathrm{~g} / \mathrm{cm}^{3}\left(1540{ }^{\circ} \mathrm{C}\right)$; retração na sinterização $17,5 \%$; tamanho médio de partícula $0,4 \mu \mathrm{m}$.

A injetora utilizada para MIBP foi uma Peltsman MIGL33 (semi-automática) [14-16]. Ela consiste de um tanque, que se conecta com um tubo direcionado para uma matriz, ambos com sistema de controle de temperatura independente. A batedeira planetária usada na mistura do pó cerâmico com os ligantes fica localizada dentro do tanque da injetora, o qual 
é conectado a uma bomba de vácuo para deaeração durante a preparação da mistura. $\mathrm{O}$ molde é fixado na abertura do tubo de saída (também aquecido) e a moldagem ocorre com a injeção de ar comprimido sobre a mistura do tanque. $\mathrm{O}$ uso de ligantes baseados em ceras com baixo ponto de fusão e baixa viscosidade possibilita o emprego de baixas pressões (da ordem de centenas de kPa) para a injeção [14-16].

A mistura usada na moldagem foi preparada diretamente na injetora e consistiu de $86 \%$ em peso de pó e $14 \%$ em peso de ligantes. Estes componentes foram misturados por $20 \mathrm{~h}$ a $90{ }^{\circ} \mathrm{C}$. Após, a mistura foi injetada em um molde metálico (a $50{ }^{\circ} \mathrm{C}$ ), como o mostrado na Fig. 2 , a $400 \mathrm{kPa}$. O molde, revestido por um filme de ptfe é levemente lubrificado com óleo de silicone e a injeção ocorre por um tempo de aproximadamente $12 \mathrm{~s}$. Após, é feita a refrigeração e a mola é retirada do molde [14-16].

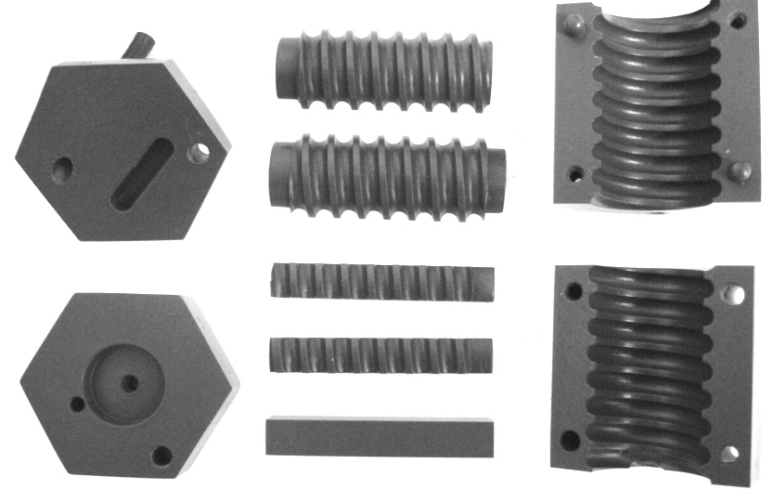

Figura 2: Molde de latão revestido com um filme de ptfe usado para injetar as molas cerâmicas em baixa pressão [8].

[Figure 2: Brass mold coated with a ptfe film used for the lowpressure injection molding of ceramic springs [8].]

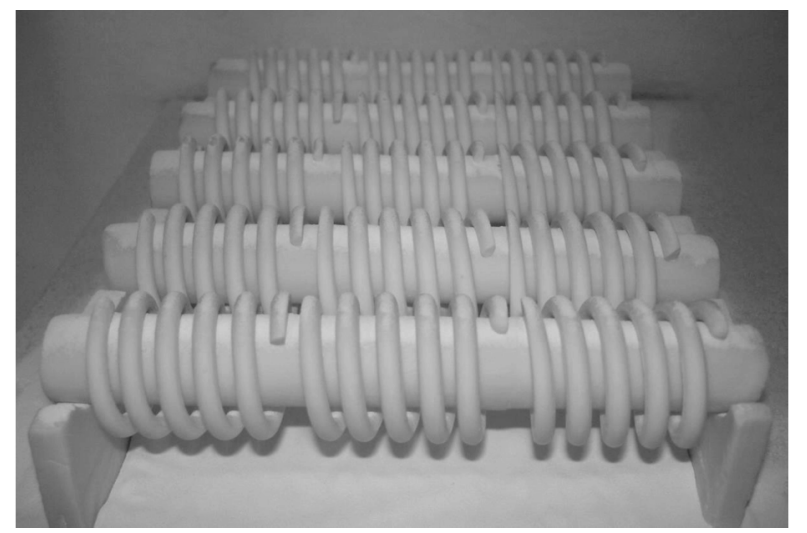

Figura 3: Molas de alumina em forno de sinterização. A mobília de forno também é de alumina e foi feita especialmente para reduzir as distorções observadas durante a sinterização das molas cerâmicas. [Figure 3: Alumina springs inside the sintering furnace. The support, also made of alumina, was produced specifically aiming to reduce distortions during the sintering of the ceramic springs.]

As molas injetadas tiveram seus ligantes removidos por aquecimento ao ar, em um recipiente contendo um leito de alumina A-1000SG, em que as peças ficam totalmente encobertas pelo pó. A queima procede neste ambiente até a temperatura de $250{ }^{\circ} \mathrm{C}$ [14-16]. Assim que os ligantes orgânicos são extraídos, as peças são pré-sinterizadas, ao ar, até uma temperatura de $1000{ }^{\circ} \mathrm{C}$. Após este tratamento, as peças podem receber acabamento, por lixamento, caso haja necessidade de eliminar algum defeito superficial existente ou melhorar o acabamento superficial [14-16]. Em seguida todas as molas são sinterizadas ao ar, apoiadas em uma mobília, também de alumina, especialmente desenvolvida para a sinterização das molas e que minimiza as distorções induzidas nesta etapa do processo. Para não aderir à mobília, esta é recoberta com uma fina camada de pó de alumina A-1 (Alcoa S.A.). A rampa de aquecimento utilizada foi de $1{ }^{\circ} \mathrm{C} /$ min até $1600^{\circ} \mathrm{C}$. A sinterização se dá, a esta temperatura, por mais duas horas [14-16]. Na Fig. 3 podemos ver as molas de alumina no forno de sinterização.

As molas cerâmicas sinterizadas obtidas tiveram as suas densidades medidas pelo método de Arquimedes.

\section{Constante de mola - fundamentação teórica}

As freqüências naturais de vibração de uma mola cilíndrica helicoidal podem ser calculadas tratando-se a mola como uma barra de seção circular reta $[17,18]$. As freqüências vibracionais $(f)$ dos modos normais longitudinais de uma barra livre com comprimento $L$ são dadas por

$$
f_{n}=\frac{n c_{S}}{2 L}
$$

na qual $n=1,2,3, \ldots$ e $c_{S}$ é a velocidade de propagação do som no sólido. Para uma barra fina, $c_{S}$ é aproximadamente igual a velocidade do som em uma barra infinita,

$$
c_{S}=\sqrt{\frac{E}{\rho}}
$$

na qual $E$ é o módulo de Young e $\rho$ é a densidade. Substituindo a equação (C) em (B) temos, para o modo fundamental ( $\mathrm{n}=1$ ),

$f=\frac{1}{2 L} \sqrt{\frac{E V}{m}}=\frac{1}{2 L} \sqrt{\frac{E A L}{m}}$

na qual $m$ é a massa e $V(=A L$, produto da área da base pela altura) é o volume do cilindro.

Em termos de módulo de Young e das suas dimensões, a constante de mola de um cilindro é dada por $K=E A / L$. Substituindo em (D), obtemos a frequência de vibração do modo longitudinal fundamental de uma barra e, consequentemente, de uma mola, dada por

$$
f=\frac{1}{2} \sqrt{\frac{K}{m}}
$$


Para a medição da constante de mola, foi montado o experimento representado esquematicamente na Fig. 4. Este sistema foi montado dentro de um forno tubular de alumina ( \pm Ø $50 \mathrm{~mm}$ ) e a frequência de vibração da mola, suspensa no interior do forno por um fio de platina, foi medida em temperaturas crescentes. A temperatura da mola é medida com o auxílio de um termopar do tipo K. A frequência de vibração da mola, $f$, é obtida percutindo a mesma com um bastão de alumina. O som é captado por um microfone que se localiza do lado externo do forno protegido com lã cerâmica. A frequência do modo longitudinal fundamental da mola é obtida a partir da análise da transformada de Fourier do sinal sonoro captado pelo microfone em função do tempo. Apesar de a mesma medição poder ser realizada utilizandose um osciloscópio digital, optou-se por registrar o sinal sonoro captado pelo microfone usando uma placa de som de um computador convencional. Neste caso, o registro do espectro sonoro emitido pela mola percutida e o cálculo da transformada de Fourier foram realizados com o auxílio do programa Visual Analyser 2011 [19]. A partir da frequência de vibração assim obtida, calcula-se a constante de mola utilizando-se a equação (E) $[17,18]$. O ensaio usando a técnica de impulso para a determinação da constante de molas cerâmicas é mais adequado que os métodos baseados na técnica de ressonância, cuja aplicação em altas temperaturas é limitada devido ao amortecimento das vibrações pela dissipação de energia na peça [20]. A mesma consideração se aplica à técnica empregada na determinação dos módulos elásticos de cerâmicas avançadas, tal como descrita na norma ASTM C 1259-08 [21].

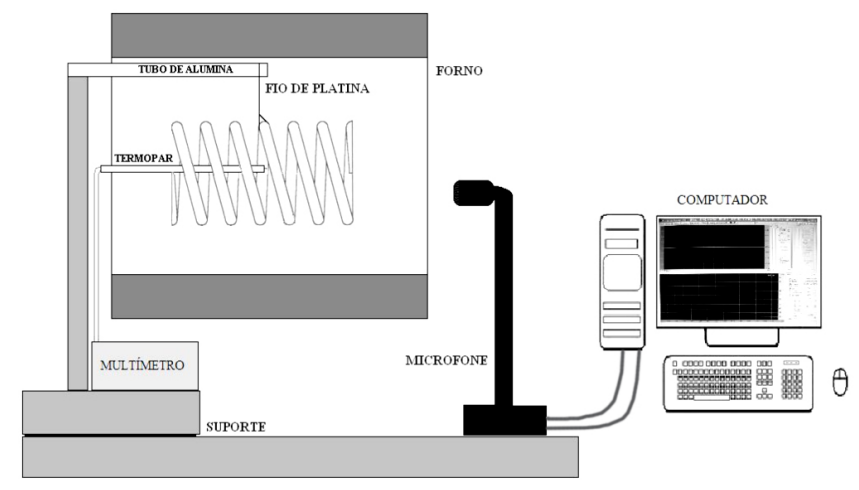

Figura 4: Esquema de montagem do experimento de medição da constante de mola.

[Figure 4: Schematics of the experimental assembly to measure the spring constant.]

\section{RESULTADOS E DISCUSSÃO}

As dimensões das molas obtidas por moldagem por injeção em baixa pressão são mostradas na Tabela I. O procedimento descrito neste trabalho para a medição da constante de mola de molas cerâmicas em alta temperatura será ilustrado com resultados obtidos com uma mola helicoidal de alumina, com uma massa de 12,66 g. A
Tabela I - Dimensões típicas das molas da alumina (com desvio padrão) produzidas por MIBP em molde de latão com revestimento de ptfe.

[Table I - Typical dimensions (and standard deviation) of the alumina springs produced by LPIM with the ptfe-coated brass mold.]

\begin{tabular}{ccccc}
\hline Material & $\begin{array}{c}\mathrm{D}_{\mathrm{o}} \\
(\mathrm{mm})\end{array}$ & $\begin{array}{c}\mathrm{d} \\
(\mathrm{mm})\end{array}$ & $\begin{array}{c}\mathrm{h} \\
(\mathrm{mm})\end{array}$ & $\mathrm{Na}$ \\
\hline Alumina & $23,08 \pm 0,05$ & $4,02 \pm 0,02$ & $28,1 \pm 0,6$ & 4 \\
\hline
\end{tabular}

$D_{o}=$ diâmetro externo das molas, $d=$ diâmetro do fio, $h=$ altura e $\mathrm{Na}=$ número de espiras ativas.

densidade da mola de alumina testada em alta temperatura, $3,90 \mathrm{~g} / \mathrm{cm}^{3}$, corresponde a $97,8 \%$ da densidade teórica [22].

$\mathrm{Na}$ Tabela II são apresentados os resultados obtidos para a mola de alumina em diferentes temperaturas. A incerteza da frequência do modo normal fundamental longitudinal da mola de alumina é de cerca de $0,2 \%$ à temperatura ambiente, o que representa uma incerteza de aproximadamente $0,4 \%$ no valor da constante de mola. A amplitude do sinal sonoro gerado pela percussão da mola no interior do forno diminui à medida que aumenta a temperatura, devido à já referida

Tabela II - Freqüências de vibração do modo longitudinal fundamental e constante de mola de uma mola de alumina em diferentes temperaturas.

[Table II - Vibrational frequency of the fundamental longitudinal mode and spring constant for an alumina spring at different temperatures.]

\begin{tabular}{ccc}
\hline $\begin{array}{c}\text { Temperatura } \\
\left({ }^{\circ} \mathrm{C}\right)\end{array}$ & $\begin{array}{c}\text { Frequência de } \\
\text { vibração }(\mathrm{Hz})\end{array}$ & $\begin{array}{c}\text { Constante de } \\
\text { mola K }\left(\mathrm{N} \mathrm{mm}^{-1}\right)\end{array}$ \\
\hline 25 & 1374 & 95,6 \\
250 & 1355 & 93,0 \\
500 & 1330 & 89,6 \\
750 & 1305 & 86,2 \\
1000 & 1285 & 83,6 \\
1100 & 1270 & 81,7 \\
\hline
\end{tabular}

dissipação da energia de vibração na mola [20]. Como conseqüência disto, a determinação da posição do pico referente ao modo longitudinal de vibração da mola na transformada de Fourier se torna progressivamente menos precisa. Ainda assim, a incerteza do valor determinado para a frequência do modo normal fundamental longitudinal da mola de alumina permanece bem inferior a $1 \%$ mesmo a $1100{ }^{\circ} \mathrm{C}$.

A Fig. 5 exibe uma imagem capturada da tela do programa usado na aquisição de dados durante o ensaio de uma mola de alumina à temperatura ambiente. O painel superior exibe o sinal sonoro (captado pelo microfone e digitalizado pela placa de som do computador) em função do tempo e o painel inferior exibe a transformada de Fourier deste sinal em tempo real. O primeiro pico (mais intenso) 


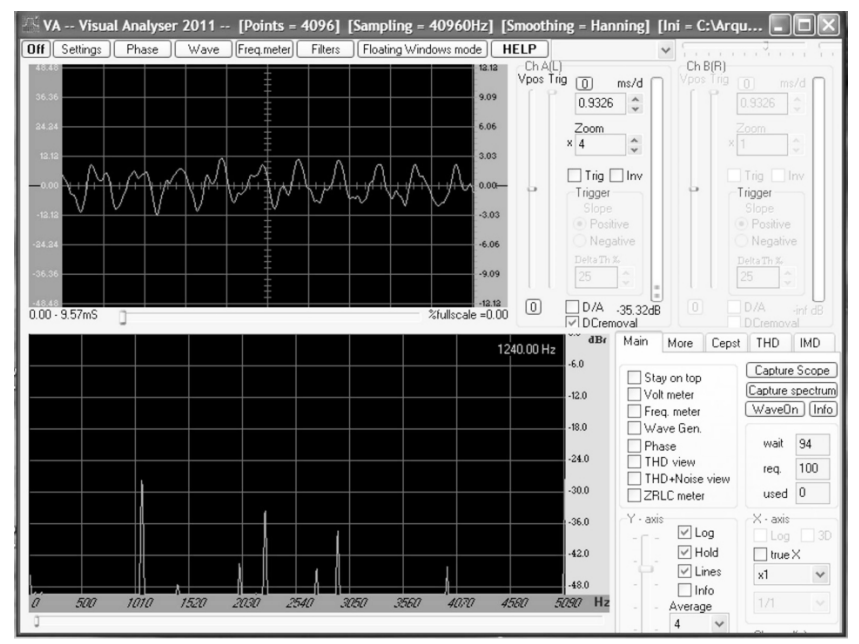

Figura 5: Tela capturada do programa Visual Analyser 2011 durante um experimento com uma mola de alumina à temperatura ambiente. O painel superior exibe o sinal sonoro captado pelo microfone em função do tempo. O painel inferior exibe a transformada de Fourier deste sinal em tempo real.

[Figure 5: Screenshot of the software Visual Analyser 2011 during an experiment carried out with an alumina spring at room temperature. The panel at the top shows the signal measured by the microphone as a function of time. The panel at the bottom shows the Fourier transform of this signal in real time.]

na transformada de Fourier, em aproximadamente $1240 \mathrm{~Hz}$, corresponde à frequência de vibração do modo longitudinal fundamental da mola cerâmica e será usado no cálculo da constante de mola.

A Fig. 6 ilustra a dependência da constante de mola com a temperatura. Na mesma figura é representada a dependência com a temperatura do módulo de cisalhamento da alumina [22]. A constante de mola e o módulo de cisalhamento

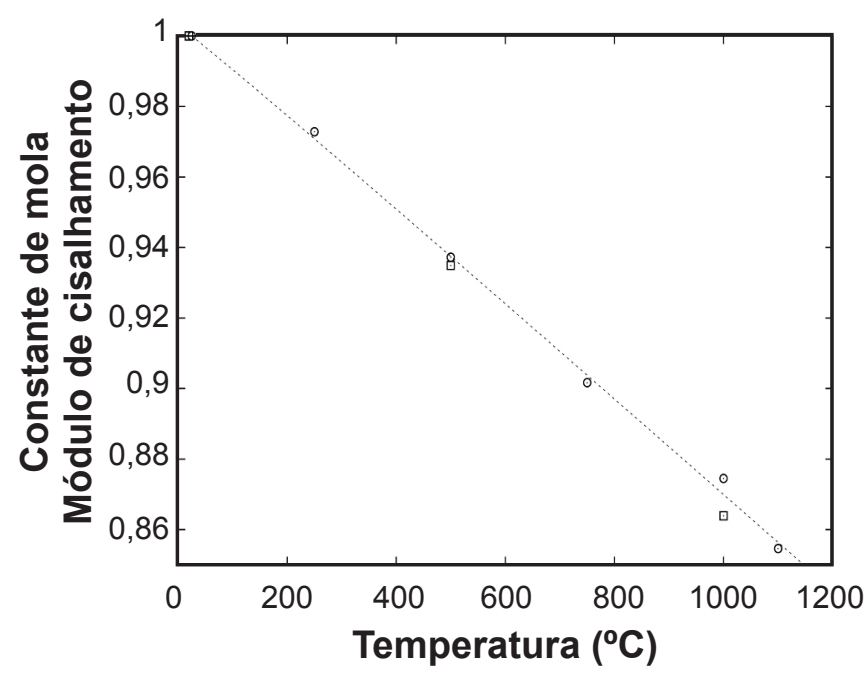

Figura 6: Constante de mola (०) e módulo de cisalhamento ( $\square$ ) da alumina, ambos normalizados em relação aos seus respectivos valores à temperatura ambiente.

[Figure 6: Spring constant (०) and alumina shear modulus ( $\square$ ), both normalized with respect to their respective room temperature values. foram ambos normalizados em relação aos seus valores à temperatura ambiente. A linha tracejada no gráfico da Fig. 6 representa o ajuste da equação de Wachtman aos valores da constante de mola em função da temperatura [23]. Pode-se observar na Fig. 6 que ambos, a constante de mola e o módulo de cisalhamento da alumina, diminuem com a temperatura. De fato, de acordo com a expressão (A) a constante de mola varia proporcionalmente ao módulo de cisalhamento $(G)$ e, portanto, o excelente acordo entre a variação com a temperatura do módulo de cisalhamento e da constante de mola, exibido na Fig. 6, corresponde ao comportamento esperado, pois o efeito da expansão térmica sobre as dimensões física da mola é relativamente pequeno. Pequenas diferenças entre o comportamento exibido pela constante de mola e pelo módulo de cisalhamento da alumina em função da temperatura podem ser devidas a fatores experimentais ou intrínsecos da mola cerâmica. De fato, tem sido reportado na literatura que o módulo de cisalhamento efetivo de uma mola cerâmica não depende apenas do material com que ela é produzida, mas também do método de conformação empregado [3]. Por isso, para aplicações tecnológicas é importante medir a constante de mola em altas temperaturas e não apenas estimar seu valor a partir da dependência com a temperatura do módulo de cisalhamento do material com que ela foi produzida.

\section{CONCLUSÕES}

Uma técnica de impulso foi utilizada para a determinação da dependência com a temperatura da frequência longitudinal fundamental de vibração de molas cerâmicas e, a partir desta, da constante de mola. Esta técnica, de fácil aplicação, utiliza recursos simples e acessíveis e permite determinar o efeito da temperatura sobre a resistência à deformação elástica de molas cerâmicas, que constitui um parâmetro fundamental em aplicações tecnológicas. Esta técnica é particularmente interessante nos casos em que a mola cerâmica é constituída de materiais ou compósitos cerâmicos cujos módulos de cisalhamento (e sua dependência com a temperatura) não sejam conhecidos com precisão. A extensão da técnica para situações que envolvam ambientes quimicamente agressivos é possível e não envolve as dificuldades inerentes a um ensaio de compressão nas mesmas condições. Evidentemente, testes de compressão ainda se fazem necessários para a determinação da tensão de ruptura de molas cerâmicas, mas a presente técnica substitui com vantagens os tradicionais ensaios de compressão na determinação da resistência à deformação elástica e no controle de qualidade de molas cerâmicas.

\section{AGRADECIMENTOS}

Este trabalho foi parcialmente financiado por FAPERGS, CAPES, FINEP e CNPq. Os autores agradecem às empresas Almatis Inc. (Leetsdale, PA, EUA) e Alcoa S.A. pelas amostras de alumina. Também agradecem a colaboração de Renan F. de Lucena Lorenzi e Cláudio A. Perottoni. 


\section{REFERÊNCIAS}

[1] J. K. Wright, R. M. Thomson, J. R. G. Evans, J. Mat. Sci. 25, 1 (1990) 149-156.

[2] W. Nakao, S. Mori, J. Nakamura, K. Takahashi, K. Ando, J. Am. Ceram. Soc. 89, 4 (2006) 1352-1357.

[3] T. Hamilton, M. Gopal, E. Atchley, J. E. Smith Jr., J. Mat. Sci. 38, 15 (2003) 3331-3335.

[4] M. L. Kaforey, C. W. Deeb, D. H. Matthiensen, J. Cryst. Growth 211, 1-4 (2000) 421-427.

[5] C. Kaya, S. Blackburn, J. Eur. Ceram. Soc. 24, 14 (2004) 3663-3670.

[6] C. Kaya, E. G. Butler, Scripta Mater. 48, 4 (2003) 359364.

[7] R. Andreola, Conformação de molas cerâmicas por moldagem por injeção em baixa pressão, Diss. Mestrado, PGMAT-UCS, RS (2007).

[8] G. Nieman, Elementos de Máquinas, V.1, Ed. Edgard Blücher, S. Paulo, SP (1971) 186-208.

[9] S. Melconiam, Elementos de Máquinas, Ed. Ática (2000) 149-163.

[10] K. Q. Qiu, Y. L. Ren, Mater. Lett. 60, 15 (2006) 18511853.

[11] I. Krindges, R. Andreola, C. A. Perottoni, J. E. Zorzi, Int. J. Appl. Ceram. Technol. 5, 3 (2008) 243-248.

[12] S. Nohut, G. A. Schneider, J. Eur. Ceram. Soc. 29, 6
(2009) 1013-1019.

[13] S. Sato, K. Taguchi, R. Adachi, M. Nakatai, Fatigue Fract. Eng. Mater. Struct. 19, 5 (1996) 529-537.

[14] J. E. Zorzi, C. A. Perottoni, J. A. H. da Jornada, Cerâmica 50, 315 (2004) 202-208.

[15] J. E. Zorzi, C. A. Perottoni, J. A. H. da Jornada, J. Mater. Sci. 37, 9 (2002) 1801-1807.

[16] J. E. Zorzi, C. A. Perottoni, J. A. H. da Jornada, Ind. Ceram. 23, 1 (2003) 47-49.

[17] S. Velasco, F. L. Román, J. A. White, Am. J. Phys. 78, 12 (2010) 1429-1431.

[18] L. Della Pietra, S. Della Valle, Meccanica 17, 1 (1982) 31-43.

[19] Disponível em http://www.sillanumsoft.org/, acessado em 28/09/2010.

[20] M. Fukuhara, I. Yamauchi, J. Mater. Sci. 28, 17 (1993) 4681-4688.

[21] ASTM C 1259-08, Standard Test Method for Dynamic Young's Modulus, Shear Modulus, and Poisson's Ratio for Advanced Ceramics by Impulse Excitation of Vibration.

[22] R. G. Munro, J. Am. Ceram. Soc. 80, 8 (1997) 19191928.

[23] J. B. Wachtman, W. R. Cannon, M. J. Matthewson, Mechanical Properties of Ceramics, $2^{\text {nd }}$ Ed., John Wiley \& Sons, Inc., NJ, EUA (2009) 48.

(Rec. 30/12/2010, Rev. 14/02/2011, Ac. 02/03/2011) 